\title{
Lack of TNF- $\alpha$-Induced MMP-9 Production and Abnormal E-Cadherin Redistribution Associated with Compromised Fusion in MCP-1-Null Macrophages
}

\author{
Eleni A. Skokos, * Antonios Charokopos, * \\ Khadija Khan, ${ }^{\dagger}$ Jackie Wanjala, $^{\dagger}$ and \\ Themis R. Kyriakides*†‡ \\ From the Departments of Pathology, ${ }^{*}$ and Biomedical \\ Engineering, ${ }^{\dagger}$ and the Interdepartmental Program in Vascular \\ Biology and Therapeutics, ${ }^{\ddagger}$ Yale University School of Medicine, \\ New Haven, Connecticut.
}

Homotypic cell fusion occurs in several cell types including macrophages in the formation of foreign body giant cells. Previously, monocyte chemoattractant protein-1 (MCP-1) was demonstrated to be required for foreign body giant cell formation in the foreign body response. The present study investigated the fusion defect in MCP-1-null macrophages by implanting biomaterials intraperitoneally in wildtype and MCP-1-null mice and monitoring the macrophage response at 12 hours to 4 weeks. MCP-1-null mice exhibited reduced accumulation and fusion of macrophages on implants, which was associated with attenuation of the foreign body response. Consistent with previous in vitro findings, the level of matrix metalloproteinase-9 (MMP-9) was reduced in MCP-1-null macrophages adherent to implants. In contrast, CCR2 expression was unaffected. In vitro studies revealed reduced tumor necrosis factor- $\alpha$ (TNF- $\alpha$ ) production and abnormal subcellular redistribution of E-cadherin and $\beta$-catenin during fusion in MCP-1-null macrophages. Exogenous TNF- $\alpha$ caused an increase in the production of MMP-9 and rescued the fusion defect. Addition of GM6001 (MMP inhibitor) or NSC23766 (Rac1 inhibitor) indicated two distinct induction pathways, one for E-cadherin/ $\beta$-catenin and one for MCP-1, TNF- $\alpha$, and MMP-9. Considered together, these observations demonstrate that induction of E-cadherin/ $\beta$-catenin is not sufficient for fusion in the absence of MCP-1 or the downstream mediators TNF- $\alpha$ and MMP-9. Moreover, attenuation of the foreign body response in intraperitoneal implants in MCP-1-null mice demonstrates that the process depends on tissue-specific factors. (Am J Pathol 2011, 178:2311-2321; DOI: 10.1016/j.ajpath.2011.01.045)
Cell-cell fusion, either homotypic or heterotypic, is critical to many biological processes including fertilization, development, bone formation, and immunity. ${ }^{1-4}$ Macrophages possess fusogenic potential and fuse among themselves and with other cell types. ${ }^{4}$ Homotypic fusion of macrophages and cells of the monocytic lineage results in formation of osteoclasts and foreign body giant cells (FBGCs). ${ }^{3}$ FBGCs are considered a hallmark of the foreign body response, which is a specialized response to implanted materials that consists of processes found in acute and chronic inflammation and wound healing. 5,6 Fusion of macrophages to form FBGCs in the proximity of biomaterials contributes to surface damage. ${ }^{5}$ Thus, strategies that limit FBGC formation and encapsulation are highly desirable.

Recent studies have shed light on the molecular mechanism of macrophage fusion including identification of surface-associated molecules such as CD44, CD47, CD200, SIRP1-a, IL-4 receptor, E-cadherin, mannose receptor, and CD36 (reviewed in Helming and Gordon ${ }^{4}$ ). In addition, blockade of intracellular molecules such as DAP12, DC-STAMP, Rac1, and STAT-6 limited macrophage fusion. ${ }^{7-10}$ Moreover, studies in monocyte chemoattractant protein-1 (MCP-1)-null and matrix metalloproteinase-9 (MMP-9)-null mice indicated a requirement for these secreted molecules in FBGC formation. ${ }^{11,12}$ Thus, it was anticipated that multiple signals converge to induce macrophage activation and "prime" the cells for fusion. In a recent study, previously reported IL-4-induced STAT-6-dependent up-regulation of E-cadherin was associated with alternative activation of macrophages. ${ }^{13}$ This study also demonstrated that IL-4 induced formation of $\mathrm{E}$-cadherin/ $\beta$-catenin complexes at

Supported by grant GM072194-01 (T.R.K.) from the National Institutes of Health.

Accepted for publication January 18, 2011.

Supplemental material for this article can be found at http://ajp. amjpathol.org or doi: 10.1016/j.ajpath.2011.01.045.

Address reprint requests to Themis R. Kyriakides, Ph.D., Department of Pathology, Yale University School of Medicine, 10 Amistad St, 301c, PO Box 208089, New Haven, CT 06519. E-mail: themis.kyriakides@yale. edu. 
the cell surface. Thus, it was envisioned that IL-4 stimulates an epithelial-like phenotype that could enable macrophages to establish intimate cell-cell contacts. However, it is unclear how formation of these contacts contributes to fusion.

Monocyte recruitment during inflammation is mediated by the action of chemokines such as MCP-1. ${ }^{14,15}$ In previous studies, MCP-1-null mice exhibited normal numbers of resident tissue macrophages and recruited similar numbers of neutrophils and eosinophils in inflammation models such as injection of thioglycollate to the peritoneum. ${ }^{14,16}$ In contrast, in the same model, monocyte recruitment in MCP-1-null mice was compromised, a finding that was also observed in mice deficient in CCR2, the only known receptor for MCP-1. ${ }^{17,18}$ Insofar as the foreign body response, MCP-1-null mice demonstrated normal monocyte recruitment after subcutaneous implantation of biomaterials and normal encapsulation. ${ }^{11}$ Differences in subcutaneous versus intraperitoneal cell recruitment in MCP-1-null mice suggested that the role of MCP-1 is tissue-specific. This suggestion is consistent with studies that demonstrated that anatomical site can greatly affect the host response to implanted materials. ${ }^{6,19}$ FBGC formation was reduced in MCP-1null mice and led to delayed biomaterial degradation. ${ }^{11}$ Subsequent fusion experiments demonstrated that MCP1-null macrophages are compromised in fusion because of inability to undergo cytoskeletal remodeling and induce MMP-9 in response to IL-4., ${ }^{8,12}$

The present study investigated the hypothesis that the foreign body response in MCP-1-null mice might be tissue-specific by implanting biomaterials intraperitoneally in wild-type (WT) and MCP-1-null mice and comparing the ensuing foreign body response. Histochemical and immunohistochemical analyses indicated a reduction in macrophage adhesion, FBGC formation, and encapsulation in MCP-1-null mice. Moreover, ultrastructural analysis suggested that these macrophages were not fully activated. To verify in vivo findings, in vitro fusion assays were performed and irregular deposition of E-cadherin and $\beta$-catenin was observed in MCP-1-null macrophages. In search of downstream mediators of MCP-1, reduced levels of the proinflammatory cytokine tumor necrosis factor- $\alpha$ (TNF- $\alpha$ ) were observed during fusion. In addition, a requirement for TNF- $\alpha$ for MMP-9 induction and macrophage fusion was demonstrated. The results suggest the presence of at least two distinct fusion pathways, one that induces expression of E-cadherin/ $\beta$-catenin and one that involves induction of MCP-1, TNF- $\alpha$, and MMP-9.

\section{Materials and Methods}

\section{Materials}

Anti-mouse macrophage antigen-3 (Mac-3) antibody (Ab) (clone M3/84), CD16/CD32 Fc blocking Ab (clone 93), anti-E-cadherin C-terminal Ab (clone 36), anti- $\beta$ catenin $\mathrm{Ab}$ (clone 14), and anti-mouse Ly-6G/LY-6C Ab (RB6-8C5) were obtained from BD Biosciences (San
Jose, CA). Anti-mouse F4/80 Ab (Cl:A3-1) was purchased from Serotec Inc. (Raleigh, NC); Rac1 inhibitor (NSC23766), from Calbiochem (Gibbstown, NJ); antimouse TNF- $\alpha$ Ab and isotype rabbit IgG, from Thermo Fisher Scientific Inc. (Rockford, IL); monoclonal antiCCR2 Ab (clone E68) and polyclonal anti-CCR2 Ab, from Abcam Inc. (Cambridge, MA); recombinant mouse (rm) granulocyte macrophage colony-stimulating factor ( $\mathrm{rm}$ GM-CSF), rm IL-4, anti-mouse MMP-9 Ab, anti-mouse E-cadherin $\mathrm{N}$-terminal $\mathrm{Ab}$, and Duoset TNF- $\alpha$ enzymelinked immunosorbent assay (ELISA) kit, from R\&D Systems, Inc. (Minneapolis, MN); rm TNF- $\alpha$, from PeproTech, Inc. (Rocky Hill, NJ); Vectastain ABC kit for IHC and Vectashield mounting medium, from Vector Laboratories, Inc. (Burlingame, CA); fluorescent dyes CSFE and PKH26 red for labeling macrophages, from Invitrogen Corp. (Carlsbad, CA) and Sigma-Aldrich Corp. (St. Louis, MO), respectively; May-Grunwald and Wright-Giemsa stains, from Sigma-Aldrich Corp.; and filters $(0.45-\mu \mathrm{m}$ pore size, mixed cellulose ester) and GM6001 (MMP inhibitor), from Millipore Corp. (Billerica, MA).

\section{Implantation of Biomaterials}

All procedures were performed in accordance with the regulations adopted by the National Institutes of Health and were approved by the Animal Care and Use Committee of Yale University. Intraperitoneal implantations were performed as described previously. ${ }^{20} \mathrm{~A}$ total of 78 MCP-1-null and 78 WT mice were used for in vivo experiments. Mice were age-matched ( 3 to 4 months) in all experiments. For implantation of filters, each mouse received one $25-\mathrm{mm}^{2}$ Millipore filter implanted in the peritoneum through a $0.7-\mathrm{cm}$ incision in the skin and peritoneum. The peritoneum was closed with sterile sutures (4.0 silk), and the skin with sterile clips. Implants were excised en bloc at 12 hours and at 1, 2, 4, 8, 14, and 28 days after implantation. At each time point, five mice per genotype were used. In addition, three WT and three MCP-1-null mice were implanted intraperitoneally with one 25-mm² ACLAR film $198 \mu \mathrm{m}$ thick (SPI Supplies, West Chester, PA) as described. Films were explanted at 48 hours and processed for transmission electron microscopy.

\section{Histologic Analysis and IHC}

Explanted biomaterials were processed and stained as described previously. ${ }^{12} \mathrm{IHC}$ was performed to detect macrophages and FBGC with the Mac-3 antibody (1: 500 ), and neutrophils with the LY-6G/LY-6C antibody (1: 250). MMP-9 was detected with an anti-MMP-9 antibody $(1.5 \mu \mathrm{g} / \mathrm{mL})$. MMP-9 was detected via antigen retrieval, which required heating the slides in citrate buffer $(\mathrm{pH} 6.0)$ for 30 minutes at $100^{\circ} \mathrm{C}$. CCR2 was detected using a polyclonal anti-CCR2 antibody (1:500). All primary antibodies were diluted in $1 \%$ bovine serum albumin in PBS. All secondary biotin-conjugated antibodies were used at 1:200 dilution. Antibodies were detected using the peroxidase reaction, and the substrate diaminobenzidine 
and nuclei were visualized using a methyl green counterstain. Sections were examined under a light microscope and photographed using an Eclipse 80i microscope with NIS-Elements D 2.30 software (both from Nikon Instruments Inc., Melville, NY).

\section{Transmission Electron Microscopy}

For transmission electron microscopy, samples were dehydrated in a graded series of ethanol, washed, and embedded in Epon. Sections 100-nm thick were examined using a Technai BioTwin EM microscope (FEI Co., Hillsboro, OR) equipped with a Morada camera (Olympus Soft Imaging Solutions GmbH, Münster, Germany).

\section{Quantitative PCR Analysis}

Mice with implants underwent lavage with $2 \mathrm{~mL}$ PBS at 2, 4 , and 7 days after implantation, and the filters and peritoneal lavage fluid were collected. The lavage fluid was centrifuged (1500 rpm for 5 minutes) and resuspended in Iscove's modified Dulbecco's medium. Cells were plated in tissue culture dishes at $37^{\circ} \mathrm{C}$ for 30 minutes. Nonadherent cells were removed, and the remaining samples, which were enriched in macrophages, were extracted for RNA using the RNeasy Mini Kit (Qiagen, Inc., Valencia, CA). Similarly, total RNA was also extracted from the filters, which contained cells that had adhered to the implant. One microgram of total RNA was translated into single-stranded cDNA using the Superscript II cDNA synthesis kit and Oligo-dT (both from Invitrogen Corp.) according to an established protocol. ${ }^{21}$ Gene expression levels were determined using the IQ5 multicolor real-time PCR and SYBR Green supermix (Bio-Rad Laboratories, Inc., Hercules, CA). Amplification of each sample was performed in duplicate. Data were normalized to the levels of the housekeeping gene GAPDH. The following mouse-specific primers were used to amplify mRNA sequences: TNF- $\alpha$ forward, 5'-CCCTCACACTCAGATCATCTTCT-3', and reverse, 5'-GCTACGACGTGGGCTACAG-3'; MMP-9 forward, 5'-CGGCACGCTGGAATGATC-3', and reverse, 5'-TCGAACTTCGACACTGACAAGAA-3'; GAPDH forward, 5'-TGGCATTGTGGAAGGGCTCATGAC-3', and reverse, 5' ATGCCAGTGAGCTTCCCGTTCAGC-3'.

For TNF- $\alpha$ and MMP-9, fold change was determined in comparison with WT samples at time 0 and 24 hours, respectively. MMP-9 mRNA was not detected at time 0 in WT samples. At each time point, five mice per genotype were analyzed, and the experiment was performed twice.

\section{In Vitro Fusion Assay}

Mouse macrophages were obtained from the bone marrow of MCP-1-null and WT mice and expanded as described previously. ${ }^{8,12}$ Expanded cells $\left(0.5 \times 10^{6}\right.$ per well) were plated on a 24-well non-tissue culture treated polystyrene plate (BD Biosciences, Franklin Lakes, NJ) in media containing $10 \mathrm{ng} / \mathrm{mL} \mathrm{rm}$ GM-CSF and $10 \mathrm{ng} / \mathrm{mL} \mathrm{rm}$ IL-4. To examine the role of TNF- $\alpha$ in fusion, in separate experiments, cells were incubated with increasing dosages $(5,10$, or $50 \mathrm{ng} / \mathrm{mL})$ of $r m$ TNF- $\alpha$. In addition, WT cells were induced to fuse in the presence of functionblocking anti-TNF- $\alpha$ antibody (5, 10 , or $20 \mu \mathrm{g} / \mathrm{mL}$ ) or isotype control $\mathrm{lgG}(20 \mu \mathrm{g} / \mathrm{mL})$. For these experiments, media, cytokines, and antibodies were changed once on day 3 , and fusion was analyzed on day 7 . In a subset of experiments, WT and MCP-1-null macrophages were fluorescently labeled with either CSFE or PKH26 red, mixed 1:1, and evaluated for fusion at day 3 as described previously. ${ }^{10}$ In addition, WT and MCP-1-null macrophages were mixed $1: 1$, and fusion was evaluated on day 7. In all fusion experiments, medium was supplemented with MMP-9-depleted serum. Cells were double-stained with May-Grunwald and Wright-Giemsa stains according to standard protocols. Macrophage fusion was determined as described previously. ${ }^{11,12}$ Experiments were performed in triplicate and repeated three times. At least 50 high-power fields were examined under a light microscope and used for quantification of macrophage fusion.

\section{Flow Cytometry}

Flow cytometry was performed on samples retrieved via peritoneal lavage at 12 hours and at 1, 2, and 8 days after implantation. Cells $\left(0.5 \times 10^{6}\right)$ were resuspended in FACS buffer ( $1 \%$ bovine serum albumin in PBS, $0.01 \%$ $\mathrm{NaN}_{3}$ ) and placed in CD16/CD32 (Fc block) for 20 minutes at $4^{\circ} \mathrm{C}$. Cells were then stained with anti-mouse F4/80 fluorescein isothiocyanate-conjugated antibody or an isotype control antibody to evaluate macrophage accumulation in the peritoneal cavity. Flow cytometry was also performed on macrophages treated with IL-4 for 24 hours in vitro. Cells were stained with a monoclonal antiCCR2 antibody (1:25) or an isotype control antibody. Stained cells were fixed in $1 \%$ formaldehyde and analyzed using a FACScan flow cytometer (BD Biosciences). Forward and side scatter gates were generated to exclude dead cells, debris, and cellular aggregates. For in vivo experiments, at each time point, five mice per genotype were used, and the experiment was performed in triplicate. For in vitro experiments, the experiment was performed twice. Results were analyzed using CellQuest software (BD Biosciences).

\section{Immunocytochemistry}

Expanded bone marrow-derived macrophages were cultured on a 24-well non-tissue culture treated plates at a density of $0.5 \times 10^{6}$ cells per well. Cells were treated with $10 \mathrm{ng} / \mathrm{mL} \mathrm{rm} \mathrm{IL-4}$ and $10 \mathrm{ng} / \mathrm{mL} \mathrm{rm}$ GM-CSF for 24, 48 , and 72 hours. In a subset of fusion experiments, macrophages were treated with $100 \mu \mathrm{mol} / \mathrm{L}$ Rac 1 inhibitor, $30 \mu \mathrm{mol} / \mathrm{L}$ GM6001, or $50 \mathrm{ng} / \mathrm{mL}$ TNF- $\alpha$. At each time point, cells were fixed and permeabilized, blocked, and incubated overnight at $4^{\circ} \mathrm{C}$ with either anti-mouse $\mathrm{E}$ cadherin, $3.5 \mu \mathrm{g} / \mathrm{mL}$, anti-mouse $\beta$-catenin (1:100), or monoclonal anti-CCR2 (1:100). Fluorescein isothiocyanate-conjugated secondary antibodies (1:100) were used for detection, and nuclei were stained with DAPI (1:1000). Cells were imaged using an Axiovert 200M microscope (Carl Zeiss Microimaging GmbH, Göttingen, Germany) equipped with fluorescent optics. 


\section{Western Blot Analysis and Zymography}

Zymography was performed on conditioned media from WT bone marrow-derived macrophages induced to fuse in the presence of $100 \mu \mathrm{mol} / \mathrm{L}$ Rac1 inhibitor or $30 \mu \mathrm{mol} / \mathrm{L}$ GM6001, and MCP-1-null macrophages induced to fuse in the presence of $50 \mathrm{ng} / \mathrm{mL} \mathrm{rm} \mathrm{TNF-} \alpha$. Conditioned media were harvested at selected time points and analyzed as described previously. ${ }^{12}$ In addition, cells were subjected to RNA extraction and analyzed using real-time quantitative PCR (qPCR). Moreover, protein cell lysates were prepared via incubation of cells with RIPA buffer and analyzed by Western blot analysis. Membranes were blotted with anti-mouse E-cadherin C-terminal antibody (1:1250) or anti-mouse $\beta$-catenin antibody (1:1000), and antibodies were detected using enhanced chemiluminescence. Equal loading of protein was determined by blotting the membranes with $\beta$-actin antibody (1:5000).

\section{Determination of TNF- $\alpha$ Concentration via ELISA}

Cell culture media were collected at 24 and 48 hours after fusing macrophages. Soluble TNF- $\alpha$ was measured in conditioned media using the sandwich Duo Set TNF- $\alpha$ immunoassay according to the supplier's instructions. All samples were run in triplicate, and the experiment was repeated twice.

\section{Statistical Analysis}

Data are given as mean (SEM) and are representative of two or more independent experiments. Student's $t$-test and analysis of variance were used to assess statistical significance. $P \leq 0.05$ was considered significant.

\section{Results}

\section{Reduced Foreign Body Response in MCP-1-Null Mice}

Histologic examination of filters implanted intraperitoneally revealed increasing accumulation of cells and extracellular matrix over time in WT mice (Figure 1). In contrast, filters in MCP-1-null mice demonstrated reduced cell accumulation, complete absence of FBGC formation, and reduced encapsulation. Cells were observed on filters in MCP-1-null mice at early time points, ie, 24 and 48 hours, which suggests that cell availability was not severely compromised. To investigate this further, recruitment of macrophages to the peritoneal cavity was analyzed by flow cytometry with F4/80 antibody. Previously, we identified the kinetics of WT peritoneal macrophage recruitment, adhesion to biomaterials, and fusion in WT mice. ${ }^{20}$ In the present study, the same method was used to analyze macrophages in the peritoneum of WT and MCP-1-null mice at 12, 24, and 48 hours and at 8 days after implantation. Analysis of lavage fluid revealed progressive accumulation of macrophages in WT and MCP-

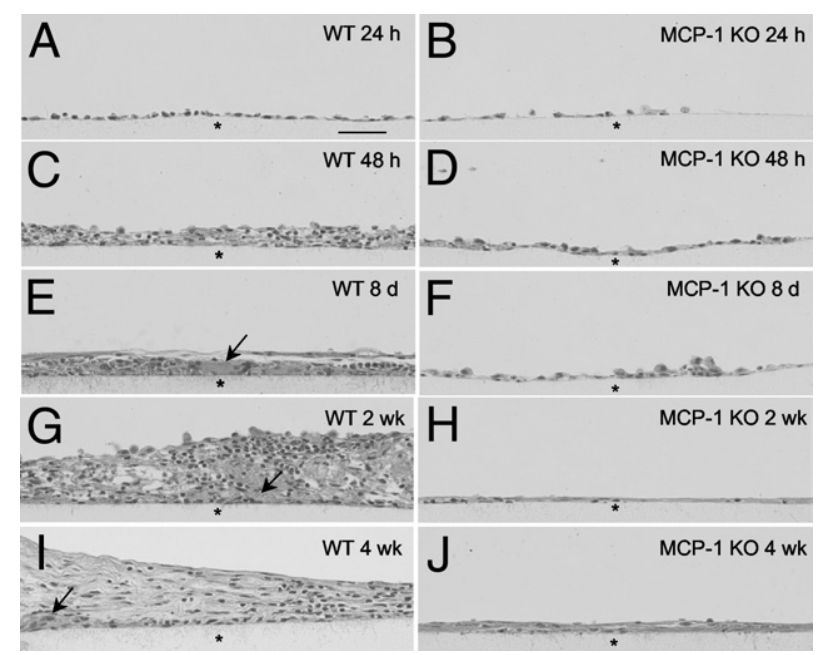

Figure 1. Reduced foreign body response in MCP-1-null mice. Representative images of sections of filters (asterisks) implanted intraperitoneally and stained with H\&E in WT (A, C, E, G, and $\mathbf{I})$ and MCP-1-null $(\mathbf{B}, \mathbf{D}, \mathbf{F}, \mathbf{H}$, and J) mice for 24 hours (A and $\mathbf{B}), 48$ hours (C and $\mathbf{D}), 8$ days ( $\mathbf{E}$ and $\mathbf{F}), 2$ weeks $(\mathbf{G}$ and $\mathbf{H}$ ), and 4 weeks (I and $\mathbf{J}$ ). Arrows indicate presence of FBGC in WT implants. At each time point, five mice per genotype were analyzed. Scale bars $=50 \mu \mathrm{m}$

1-null mice, which was not significantly different between the two groups (see Supplemental Figure S1 at $h$ ttp://ajp. amjpathol.org). Filters implanted intraperitoneally in MCP1-null mice were not fully encapsulated at 4 weeks (Figure $1 \mathrm{~J})$. Estimation of mean (SEM) capsule thickness at this time point revealed a significant reduction in MCP1-null mice $(5.7 \pm 1.8 \mu \mathrm{m})$ compared with WT (68.6 \pm $18.9 \mu \mathrm{m}) ; n=5, P \leq 0.01$.

\section{Effect of MCP-1 Deficiency on Macrophage Recruitment and Adhesion}

Macrophage adhesion and accumulation on filters were assessed by $\mathrm{IHC}$ using Mac-3 antibody, and revealed the presence of numerous macrophages on WT implants and reduced long-term accumulation on MCP-1-null implants (Figure 2). Analysis of short-term (12 and 24 hours) implants demonstrated similar macrophage adhesion to implants in WT and MCP-1-null mice, confirming that MCP1-null mice have a normal resident and elicited peritoneal macrophage population (Figure 2, A-D). Consistent with findings of histologic analysis, the presence of adherent macrophages in MCP-1-null mice did not increase significantly over time (Figure 2, H, J, L, and N). Moreover, Mac3-positive FBGCs were not observed on implants from these mice. Immunohistochemical detection of neutrophils was also performed to further evaluate the inflammatory response, and the presence of these cells was equal in implants from WT and MCP-1-null mice (see Supplemental Figure S2 at http://ajp.amjpathol.org). The antibody used to detect neutrophils, LY-6G/LY-6C, can detect some subpopulations of monocytes; however, in the present experiments, it did not interact with the bulk of implant-adherent macrophages. ${ }^{22}$ 


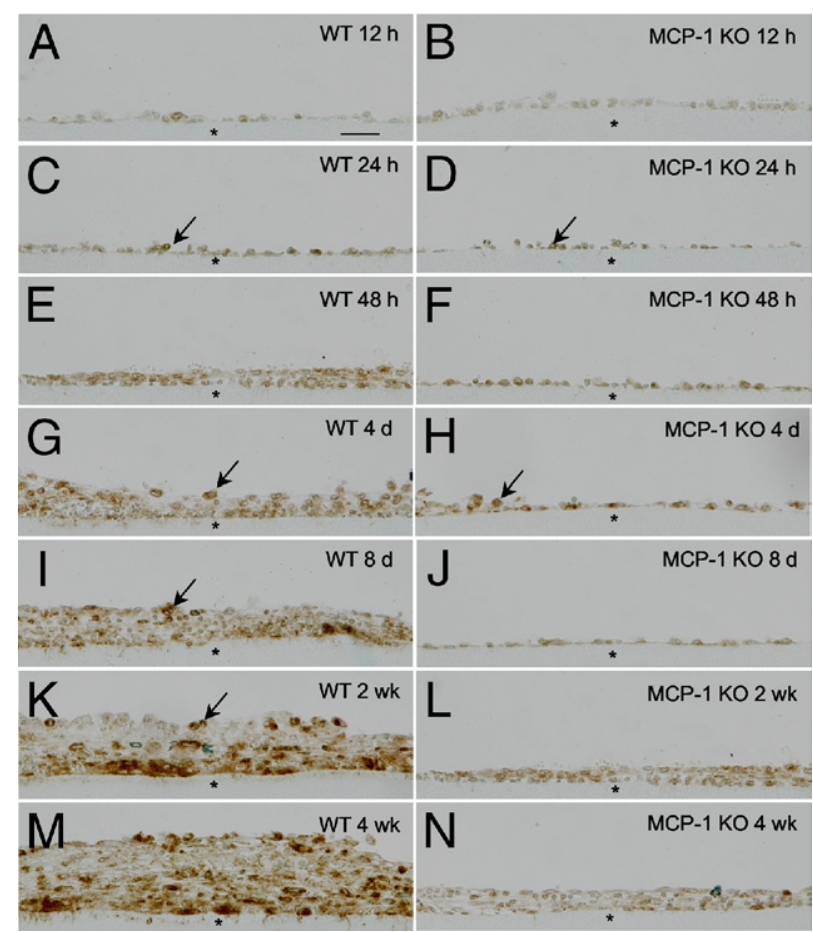

Figure 2. Reduced macrophage accumulation in MCP-1-null mice. Representative images of filters (asterisks) implanted intraperitoneally in WT (A $\mathbf{C}, \mathbf{E}, \mathbf{G}, \mathbf{I}, \mathbf{K}$, and $\mathbf{M})$ and MCP-1-null $(\mathbf{B}, \mathbf{D}, \mathbf{F}, \mathbf{H}, \mathbf{J}, \mathbf{L}$, and $\mathbf{N})$ implants for 12 hours $(\mathbf{A}$ and $\mathbf{B}), 24$ hours $(\mathbf{C}$ and $\mathbf{D}), 48$ hours $(\mathbf{E}$ and $\mathbf{F}), 4$ days $(\mathbf{G}$ and $\mathbf{H}), 8$ days (I and $\mathbf{J}), 2$ weeks ( $\mathbf{K}$ and $\mathbf{L}$ ) and 4 weeks $(\mathbf{M}$ and $\mathbf{N})$ and stained with Mac-3 antibody. Arrows indicate presence of macrophages. Immunoreactivity was detected using the peroxidase reaction (brown), and nuclei were counterstained with methyl green. At each time point, five mice per genotype were analyzed. Scale bar $=50 \mu \mathrm{m}$

\section{MCP-1-Null Macrophages Exhibit \\ Ultrastructural Abnormalities and Express Low Levels of MMP-9}

Transmission electron microscopy was performed to evaluate the morphologic features of macrophages adherent on ACLAR film in vivo at 48 hours. Unlike WT macrophages, which demonstrated evidence of activation, MCP-1-null macrophages did not exhibit membrane extensions, were smaller, and did not show extensive spreading (Figure 3). Based on previous observation of reduced MMP-9 induction in MCP-1-null macrophages in an in vitro fusion assay, ${ }^{12}$ it was wondered whether expression of this gelatinase was reduced in vivo. Immunohistochemical detection of MMP-9 revealed reduced levels in cells adherent to implants in MCP-1-null mice (Figure 3, D-M). To quantify expression of MMP-9, qPCR analysis was performed on RNA isolated from cells recovered via peritoneal lavage or from cells attached to the implant at 2, 4, and 7 days after implantation. For comparative analysis, the expression level of MMP-9 in WT lavage macrophages at 2 days was given a value of 1 and was used for comparison with all other conditions. This time point was selected as baseline because resident peritoneal macrophages did not express MMP-9 (data not shown). Overall, MMP-9 expression in WT mice was higher in implant-adherent cells and increased over time (Figure $3 \mathrm{~N}$ ), which suggests that interaction with the implant induced an increase in MMP-9. Compared with WT cells, MCP-1-null adherent cells demonstrated lower MMP-9 expression. In addition, over time, MMP-9 expression in MCP-1-null cells did not increase to a similar extent as in WT cells.

\section{MCP-1-Null Macrophages Exhibit Irregular Deposition of E-Cadherin and $\beta$-Catenin}

Recent studies involving IL-4-induced in vitro macrophage fusion have implicated induction of E-cadherin as a requirement for fusion. ${ }^{9,10,13}$ To further characterize the participation of E-cadherin in fusion, its expression and distribution were detected by Western blot analysis and immunocytochemical analysis of bone marrow-derived macrophages, respectively. Both WT and MCP-1-null macrophages treated with IL-4 exhibited induction of Ecadherin at 24 and 48 hours, and both expressed more at 48 hours (Figure 4G). Immunofluorescent detection revealed a membrane-associated pattern at 24 hours and a shift to cytoplasmic accumulation at 48 hours (Figure 4, A
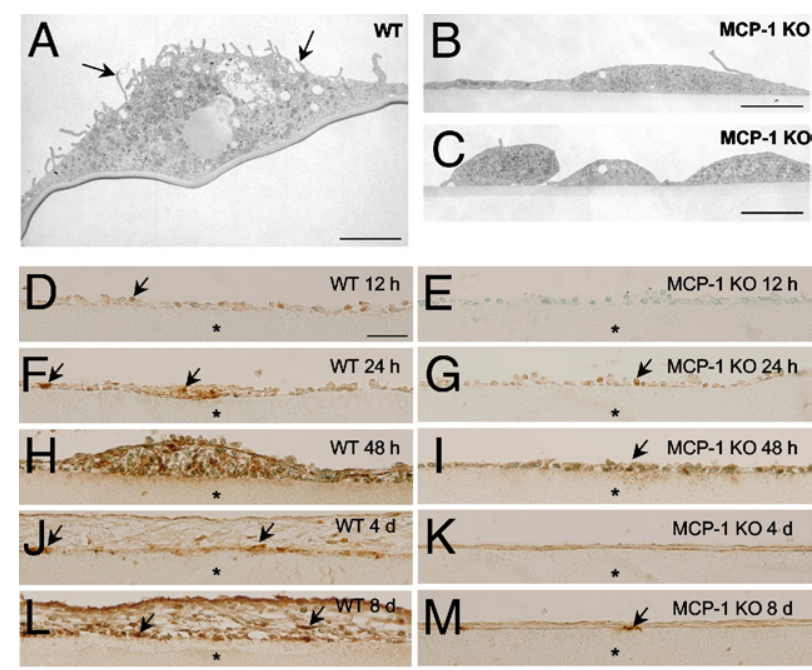

$\mathrm{N}$

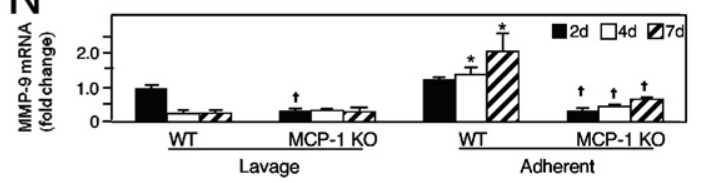

Figure 3. Abnormal macrophage activation and reduced MMP-9 expression during the foreign body response in MCP-1-null mice. Representative TEM images of WT (A) and MCP-1-null macrophages (B and C) on ACLAR film implanted for 48 hours. The MCP-1-null macrophages lack the hallmarks of activation. Three mice per genotype were analyzed. Representative images of filters (asterisks) implanted intraperitoneally in WT ( D, F, H, J, and $\mathbf{L}$ ) and MCP-1-null (E, G, I, K, and $\mathbf{M}$ ) implants for 12 hours (D and $\mathbf{E}), 24$ hours (F and $\mathbf{G}), 48$ hours $(\mathbf{H}$ and $\mathbf{I}), 4$ days $(\mathbf{J}$ and $\mathbf{K})$, and 8 days (L and $\mathbf{M})$ and stained with anti-MMP-9 Ab. Arrows indicate presence of MMP-9-positive macrophages and FBGC. Immunoreactivity was detected using the peroxidase reaction (brown), and nuclei were counterstained with methyl green. Five mice per genotype were analyzed. N: MMP-9 gene expression of nonadherent (lavage) and filter-adherent cells at 2, 4, and 7 days after implantation. At each time point, five mice per genotype were used. Analysis was performed in triplicate. Results are given as mean (SEM) fold change and are representative of two independent experiments that were normalized relative to the housekeeping gene GAPDH $(n=5) .{ }^{*}$ and $\dagger$ indicate $P \leq 0.05$ when comparing lavage and adherent cells and between WT and MCP-1-null, respectively. Scale bars: $10 \mu \mathrm{m}(\mathbf{A}-\mathbf{C}) ; 50 \mu \mathrm{m}(\mathbf{D}-\mathbf{M})$. 

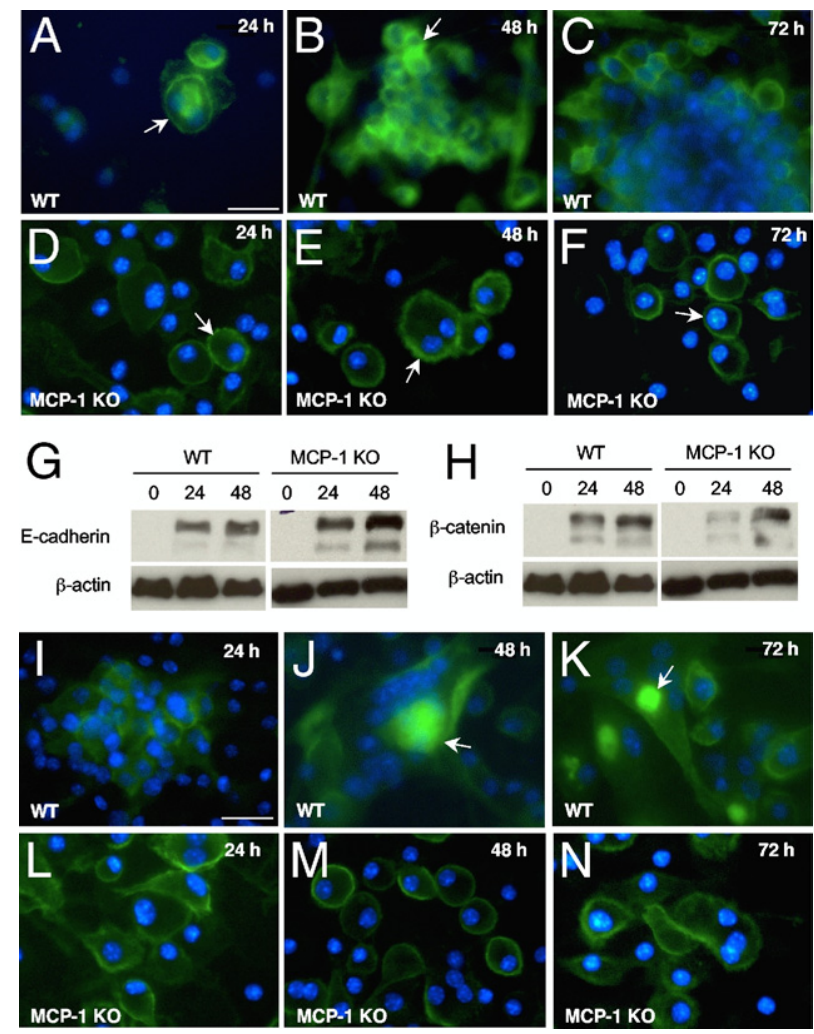

MCP-1 KO

Figure 4. Induction and redistribution of E-cadherin and $\beta$-catenin during macrophage fusion. Representative images of macrophages from WT (A, B $\mathbf{C}, \mathbf{I}, \mathbf{J}$, and $\mathbf{K})$ and MCP-1-null $(\mathbf{D}, \mathbf{E}, \mathbf{F}, \mathbf{L}, \mathbf{M}$, and $\mathbf{N})$ mice cultured in the presence of IL- 4 and GM-CSF for 24 hours $(\mathbf{A}, \mathbf{D}, \mathbf{I}$ and $\mathbf{L}), 48$ hours $(\mathbf{B}, \mathbf{E}, \mathbf{J}$, and $\mathbf{M}$ ), and 72 hours $(\mathbf{C}, \mathbf{F}, \mathbf{K}$, and $\mathbf{N})$. E-cadherin $(\mathbf{A}-\mathbf{F})$ and $\beta$-catenin $(\mathbf{I}-\mathbf{N})$ were detected by immunocytochemistry using FITC (green), and nuclei were stained with DAPI (blue). Arrows indicate E-cadherin in a membraneassociated pattern (A-F) and accumulation of $\beta$-catenin in cytoplasmic pools (I-N). Scale bars: $25 \mu \mathrm{m}(\mathbf{A}-\mathbf{F}$ and $\mathbf{I}-\mathbf{N})$. $\mathbf{G}$ and $\mathbf{H}$ : Cell lysates of macrophages induced to fuse for 24 and 48 hours from WT and MCP-1-null mice were analyzed using Western blot analysis for E-cadherin and $\beta$-catenin expression. Equal loading was determined by detection of $\beta$-actin. All experiments were repeated three times.

and B). At 72 hours, E-cadherin distribution in WT cells was diffuse in the cytoplasm of fused cells but was retained in the membranes of single cells (Figure 4C). MCP-1-null macrophages displayed a pattern consistent with membrane association throughout the duration of the experiment (Figure 4, D-F).

E-cadherin associates with $\beta$-catenin at the cell membrane, and this interaction regulates the activity of $\beta$-catenin. ${ }^{23,24}$ Moreover, E-cadherin association with $\beta$-catenin during IL-4-induced macrophage fusion has been demonstrated. ${ }^{13}$ It was wondered whether expression and distribution of $\beta$-catenin in WT and MCP1-null macrophages mirrored that of E-cadherin. Examination of $\beta$-catenin levels by Western blot analysis revealed that it was induced similarly in WT and MCP-1null macrophages and with a temporal pattern similar to that of E-cadherin (Figure $4 \mathrm{H}$ ). At 24 hours, the levels of $\beta$-catenin were somewhat reduced in MCP-1-null compared with WT macrophages. To expand on these observations, immunocytochemical analysis of $\beta$-catenin distribution in cells undergoing fusion was performed, and a membrane-associated pattern was observed in WT and
MCP-1-null cells at 24 hours (Figure 4, I and L). In WT fused cells, $\beta$-catenin was localized in cytoplasmic pools at 48 and 72 hours (Figure $4, \mathrm{~J}$ and K). DAPI staining demonstrated that these pools were not associated with nuclei. Moreover, co-localization of E-cadherin and $\beta$-catenin in WT fused cells was limited (data not shown). In contrast to WT macrophages, $\beta$-catenin in MCP-1-null macrophages at 48 and 72 hours remained in a membrane-associated pattern (Figure 4, M and N).

\section{Inhibition of Rac-1 Leads to Reduction in MMP-9 Expression}

Rac1 activation is an indispensable early step in FBGC formation. ${ }^{8}$ Moreover, MCP-1-null macrophages were deficient in IL-4-induced activation of Rac1, and inhibition of Rac1 with the low-molecular-weight inhibitor NSC23766 attenuated FBGC formation in vitro and in vivo. In addition, in a separate study, lack of MMP-9 induction by MCP-1-null macrophages was observed under fusogenic conditions. ${ }^{12}$ To expand on these observations, the effect of Rac1 inhibition on IL-4-induced MMP-9 expression in WT cells was determined, and it was completely abolished (Figure 5A). In contrast, this inhibitor did not
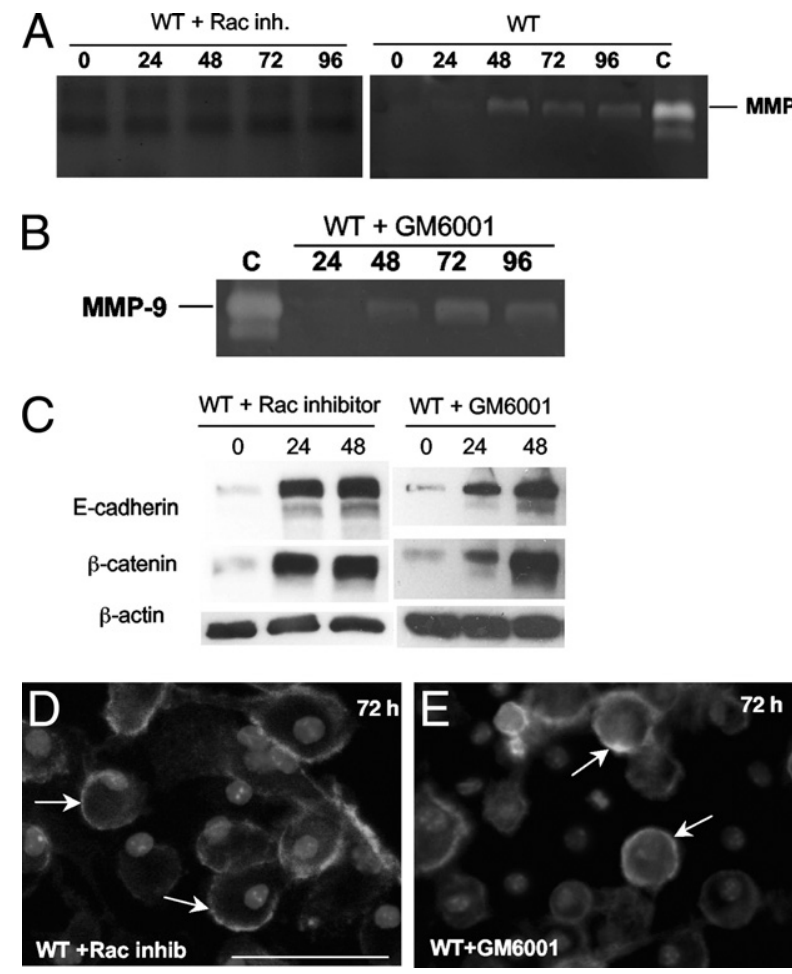

Figure 5. Inhibition of Rac1 or MMPs does not alter E-cadherin expression but enhances retention at the cell surface. A and $\mathbf{B}$ : WT macrophages were induced to fuse in the presence of either Rac1 inhibitor NSC23766 or the MMP inhibitor GM6001. Media samples were collected at 24, 48, 72, and 96 hours and were analyzed by gelatin zymography. rm MMP-9 was used as a positive control (lane C in $\mathbf{A}$ and $\mathbf{B}$ ). C: Cell lysates were prepared at 0, 24, and 48 hours from the same treatment groups, and were analyzed by Western blot analysis for E-cadherin and $\beta$-catenin expression. Equal loading was determined by $\beta$-actin. $\mathbf{D}$ and $\mathbf{E}$ : Distribution of E-cadherin in WT macrophages at 72 hours during inhibition of Rac1 or MMPs. Nuclei were stained with DAPI. Arrows indicate retention of E-cadherin in a membrane-associated pattern. Scale bar: $25 \mu \mathrm{m}$ (D and $\mathbf{E}$ ). All experiments were repeated twice. 

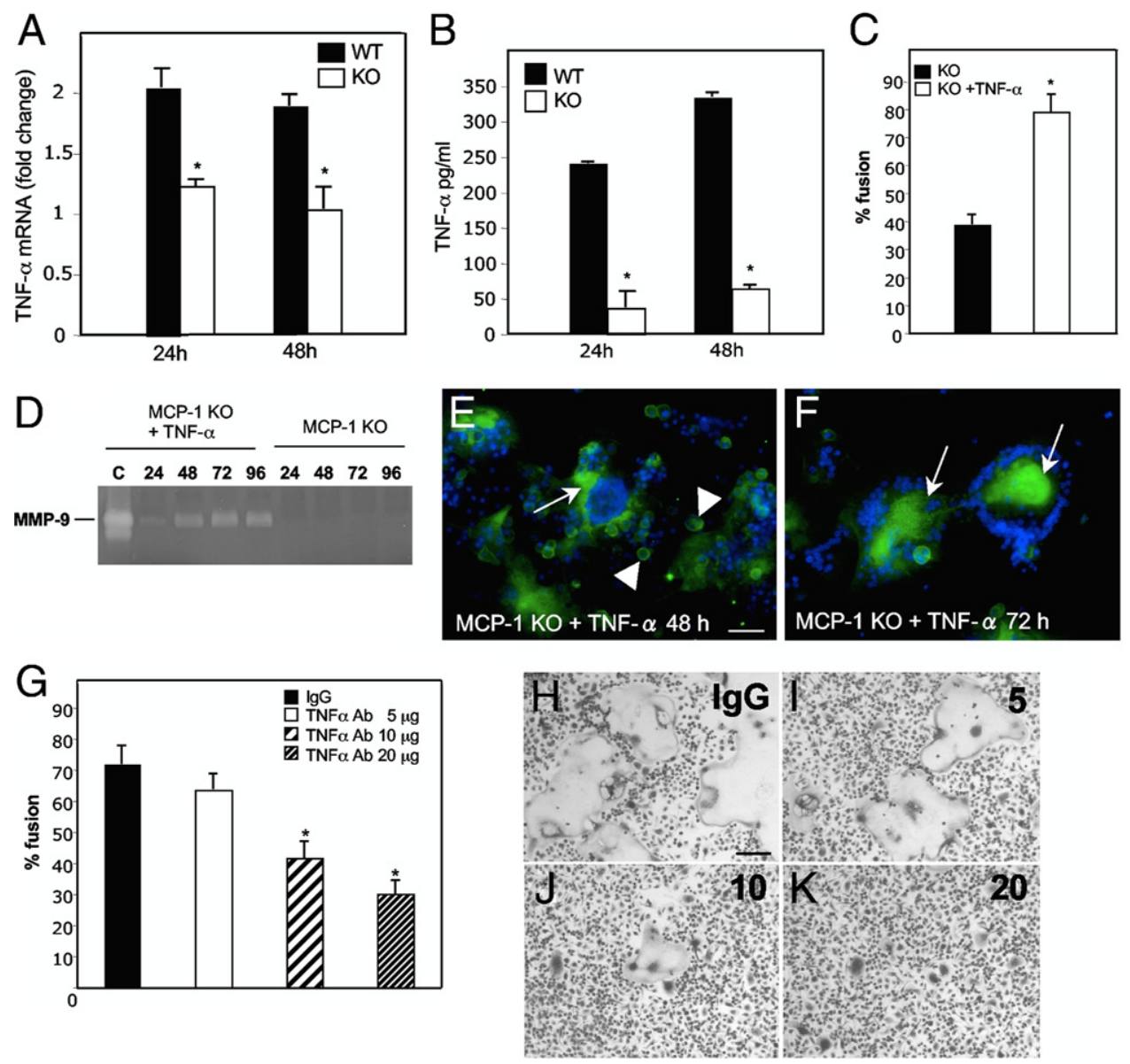

Figure 6. TNF- $\alpha$ induced MMP-9 expression is required for macrophage fusion. A: TNF- $\alpha$ gene expression was evaluated during fusion using RT-PCR, and was reduced in MCP-1-null macrophages. Results are given as mean (SEM) fold change and are representative of two independent experiments that were normalized relative to the housekeeping gene GAPDH $(n=5)$. B: Similarly, enzyme-linked immunosorbent assay analysis of conditioned media revealed reduced levels of TNF- $\alpha$ $(n=5)$. C: Quantification of fusion was performed via analysis of images double-stained with May-Grunwald and Wright-Giemsa. Percentage of fusion was determined by enumerating nuclei in FBGC in relation to total number of nuclei $(n=3)$. D: Conditioned media from MCP-1-null macrophages undergoing fusion in the presence or absence of TNF- $\alpha$ for $24,48,72$, and 96 hours were analyzed by gelatin zymography. $\mathbf{E}$ and F: E-cadherin was detected by immunocytochemistry using FITC (green), and nuclei were stained with DAPI (blue). Arrows indicate E-cadherin accumulation in the cytoplasm. Arrowheads indicate membrane-associated E-cadherin. G: Dose-dependent inhibition of WT macrophage fusion by anti-TNF- $\alpha$ antibody. Representative images of macrophages induced to fuse in the presence of isotype $\operatorname{IgG}(\mathbf{H})$ at 5 (I), $10(\mathbf{J})$, or $20(\mathbf{K}) \mu \mathrm{g}$ anti-TNF- $\alpha$ antibody $(n=3)$. Scale bars: $25 \mu \mathrm{m}(\mathbf{E}$ and $\mathbf{F}) ; 100 \mu \mathrm{m}(\mathbf{H}-\mathbf{K}) .{ }^{*} P \leq 0.05(\mathbf{A}-\mathbf{C})$. All experiments were performed at least twice.

block expression of E-cadherin or $\beta$-catenin (Figure $5 \mathrm{C}$ ). Similarly, GM6001, an inhibitor of MMP activity that also inhibits macrophage fusion, caused a delay in expression of E-cadherin and $\beta$-catenin at 24 hours but did not have a significant effect at 48 hours (Figure 5C). It was postulated that these inhibitors, similar to the MCP-1 deficiency, might alter the distribution pattern but not the levels of E-cadherin. To examine this hypothesis, the distribution of E-cadherin in cells treated with either inhibitor during fusion was analyzed, and it was observed that it was predominantly in a membrane-associated pattern and did not show accumulation in the cytoplasm (Figure 5, D and E).

\section{TNF- $\alpha$-Induced MMP-9 Expression Is Required for Macrophage Fusion}

TNF- $\alpha$ induces expression of MMP-9 in a number of inflammatory settings; however, its participation in macrophage fusion has not been reported. ${ }^{25-27}$ It was hypothesized that changes in TNF- $\alpha$ levels in MCP-1-null macrophages during fusion were contributing to the fusion defect. qPCR analysis of WT and MCP-1-null bone marrow macrophages undergoing fusion at 24 and 48 hours revealed a significant reduction in TNF- $\alpha$ in the MCP-1-null macrophages (Figure 6A). For comparative analysis, the level of expression of TNF- $\alpha$ in untreated WT macrophages was given a value of 1 and was used for comparison with all other conditions. Moreover, enzymelinked immunosorbent assay analysis of conditioned medium at 24 and 48 hours revealed a dramatic decrease in TNF- $\alpha$ levels in MCP-1-null macrophages (Figure 6B). To further characterize the effect of reduced $\mathrm{TNF}-\alpha$ on MCP-1 deficiency, the ability of MCP-1-null macrophages to fuse in the presence of exogenous TNF- $\alpha$ (5, 10 , and $50 \mathrm{ng} / \mathrm{mL}$ ) was determined (see Supplemental Figure S3 at http://ajp.amjpathol.org). Enumeration of fusion of MCP-1-null macrophages in the presence of $\mathrm{TNF}-\alpha$ revealed an increase in overall fusion to $>75 \%$, similar to that of WT macrophages (Figure 6C). In addition, analysis of conditioned media at zymography revealed restoration of MMP-9 expression (Figure 6D). Immunocytochemical analysis of MCP-1-null macrophages undergoing fusion in the presence of TNF- $\alpha$ at 48 and 72 hours revealed loss of membrane-associated E-cadherin in fused cells (Figure 6, E and F). Thus, addition of TNF- $\alpha$ to MCP-1-null macrophages during fusion restores MMP-9 levels, induces redistribution of E-cadherin, and rescues the fusion defect in these cells. To confirm the requirement for TNF- $\alpha$ in macrophage fusion, WT macrophage fusion was evaluated in the presence of an anti-TNF- $\alpha$ function-blocking antibody, and was observed to be reduced in a dosedependent manner (Figure 6, G-K). This effect was not observed with addition of isotype IgG. 


\section{Normal CCR2 Levels and Induction in MCP-1-Null Macrophages}

It has been suggested that exposure of macrophages to IL-4 in vitro induces an activation subtype characterized as alternative, which is thought to be typical of macrophages that participate in tissue repair. ${ }^{28} \mathrm{CCR} 2$, the receptor for MCP-1, can be induced during macrophage activation, and influences their specification. Analysis of its expression in vivo and in vitro demonstrated similar levels for WT and MCP-1-null cells (see Supplemental Figure S4 at http://ajp.amjpathol.org). Specifically, immunohistochemical analysis of implantadherent cells revealed similar levels in WT and MCP1-null mice. Consistent with the in vivo findings, in vitro immunofluorescence and flow cytometry revealed equal induction of CCR2 after exposure of WT and MCP-1-null macrophages to IL-4 for 24 hours.

\section{WT Macrophages Rescue MCP-1-Null Macrophages}

The present findings suggested that MCP-1-null macrophages were capable of inducing E-cadherin and $\beta$-catenin but were deficient in producing TNF and secreting MMP-9. We examined whether in a co-incubation setting, WT macrophages could rescue fusion in MCP-1null macrophages. Co-incubation of differentially labeled WT and MCP-1 macrophages at a ratio of 1:1 revealed extensive fusion between the two populations at day 3 (see Supplemental Figure S5 at http://ajp.amjpathol.org). The experiment was repeated with unlabeled WT and MCP-1-null macrophages mixed at a ratio of $1: 1$, and fusion was quantified on day 7 and observed to be similar to that of WT macrophages.

\section{Discussion}

A series of implant experiments was performed in the peritoneal cavity of MCP-1-null and WT mice, and normal macrophage recruitment and initial adhesion to biomaterials were observed. However, after day 2, the accumulation of macrophages on the implant surface in MCP-1null mice was compromised and associated with reduced encapsulation over the long term. This observation was striking, and represents the first demonstration of almost complete attenuation of the foreign body response in a genetic mouse model. These findings are consistent with those of previous studies that described reduced acute inflammatory responses in the peritoneum in MCP-1-null mice. More important, they demonstrate that the foreign body response can be tissue-specific because the MCP-1-null mice exhibited different outcomes after intraperitoneal versus subcutaneous implantation. Based on their reduced accumulation, ultrastructural appearance, and compromised induction of MMP-9, it was hypothesized that MCP-1-null macrophages fail to undergo proper activation during the foreign body response intraperitoneally. Additional evidence for deregulation of MCP-1-null macrophages was provided in a wound study in which these cells exhibited altered chemokine expression including higher levels of IL-1 $\beta$, MIP-1, and IL-11. ${ }^{29}$ Thus, it is possible that in the context of the peritoneal foreign body response, MCP-1-null macrophages undergo abnormal activation, leading to attenuation of the foreign body response.

IL-4 induces alternative activation, and it has been proposed that alternative activation leads to induction of fusogenic molecules. ${ }^{28}$ Moreover, a recent study has linked E-cadherin induction with alternative activation and assumption of an epithelial-like phenotype by macrophages before fusion. ${ }^{13}$ Thus, it is tempting to speculate that macrophages undergo alternative activation in the foreign body response. However, the concept of macrophage activation has expanded based on the theory that macrophages can exhibit overlapping gradients of activation including classical, alternative, and immunoregulatory activation. ${ }^{30}$ For example, in the present study, induction of TNF- $\alpha$, typically associated with classical activation, was observed after IL-4 treatment. In addition, IL-4 induced CCR2 expression that was normal in MCP-1-null macrophages. Considered together, these observations suggest that biomaterial-induced macrophage activation involves aspects of all subtypes and that the combination represents a uniquely activated macrophage that is specific for the foreign body response. Consistent with this suggestion, a recent study demonstrated that wound macrophages exhibited a complex phenotype that included features associated with both alternative and classical activation. ${ }^{31}$

MMP-9 is expressed during human macrophage fusion in vitro. ${ }^{32}$ Expression of MMP-9 in vitro during the fusion of peritoneal macrophages has also been reported. ${ }^{10}$ Studies in MMP-9-null mice suggested that this enzyme is critical for macrophage fusion and that it could also modulate several aspects of the foreign body response. ${ }^{12}$ In addition, in the same study it was observed that MCP-1-null macrophages expressed low levels of MMP-9 during fusion in vitro. The present study expanded on these findings by showing compromised induction of MMP-9 in vivo in MCP-1-null mice. It is unclear whether MCP-1 modulates MMP-9 expression directly. However, in a model of bleomycin-induced pulmonary fibrosis, it was suggested that MCP-1 might regulate MMP-9 expression; however, no direct evidence was provided. ${ }^{33}$ In addition, reduced expression of MMP-9 in MCP-1-null macrophages can be restored by addition of TNF- $\alpha$, an acute-phase proinflammatory cytokine with a role in the foreign body response that has received little attention. ${ }^{6,34}$ TNF- $\alpha$ also induces apoptosis in macrophages adherent to biomaterials, and IL-4 inhibits the apoptosisinducing abilities of TNF- $\alpha .{ }^{21,35}$ More relevant to this study, Robinson et $\mathrm{al}^{36}$ demonstrated that in the monocytic cell line THP-1 and in human peripheral blood monocytes, addition of rMCP-1 stimulated TNF- $\alpha$ expression, which in turn induced MMP-9 release. In addition, those authors demonstrated that by inhibiting the activity of TNF- $\alpha$, reduced expression of MMP-9 was observed. However, the mechanism through which MMP-9 expression was regulated was not described. In the macrophage cell line, RAW 264.7, MMP-9 expression was reg- 
ulated at the transcriptional level by nuclear factor $-\kappa \mathrm{B},{ }^{37}$ which is a major target of TNF-R1 activation (reviewed in Bianchi and Meier ${ }^{38}$ ). Recently, TNF- $\alpha$ was demonstrated to induce MMP-9 expression in lung epithelial cells via activation of the TRAF2/PKC- $\alpha$ pathway. ${ }^{39}$ It is unclear whether the same mechanism also participates in induction of MMP-9 in macrophages. Moreover, TNF- $\alpha$ is involved in fusion of tartrate-resistant acid phosphatase-positive mononuclear cells into multinuclear cells in a RANK (receptor activator of nuclear factor- $\kappa \mathrm{B}$ ) ligand-independent manner. ${ }^{40}$ In the present system, TNF- $\alpha$ did not induce fusion in the absence of IL-4 (data not shown), which suggests that bone marrow-derived monocytes require additional signals for fusion. However, blockade of TNF- $\alpha$ inhibited fusion, which, to our knowledge, confirms for the first time the requirement for this cytokine in this process.

The search for putative MMP-9 substrates whose cleavage might be necessary for fusion led to investigation of E-cadherin. Previously, analysis of human macrophage lysates indicated that expression of E-cadherin is induced during fusion. 9,10,13 Because E-cadherin participates in formation of tight junctions, its involvement in fusion seemed counterintuitive. Based on the present findings, it was speculated that macrophage fusion requires MMP-9 to disrupt the extracellular interactions of E-cadherin. MMP-9-mediated E-cadherin ectodomain shedding has been demonstrated in tumor cells. ${ }^{41}$ Analysis of E-cadherin distribution at immunofluorescence revealed a striking and dynamic redistribution pattern that was initially induced in a membrane-associated pattern followed by accumulation in the cytoplasm. In a temporal context, the switch of E-cadherin localization from membrane to cytoplasm coincided with the onset of MMP-9 expression. Because of the profound changes in cytoskeletal architecture of macrophages undergoing fusion, it was postulated that E-cadherin expression and redistribution from the cell membrane to the cytoplasm are mediated by cellular remodeling. It is unclear whether the redistribution of $\mathrm{E}$-cadherin and $\beta$-catenin are required for fusion per se or represent a fusion-associated event. However, studies have demonstrated dramatic changes in subcellular localization and a unique cytoplasmic deposition pattern for $\beta$-catenin. Moreover, the importance of E-cadherin in fusion has been demonstrated in studies in which an E-cadherin functionblocking antibody limited IL-4-induced fusion. ${ }^{9}$ Thus, it must be suggested that these changes are not mere associations to the fusion process.

Based on studies in genetically modified mice, it is now appreciated that IL-4 induces fusogenic signals that involve the IL-4-receptor, the signaling adaptor DAP12, the transmembrane receptor DC-STAMP, and the signaling molecule TREM-2 (Figure 7) (reviewed in Helming and Gordon $^{4}$ ). In this context, IL-4 stimulates the JAK/STAT pathway (JAK1/JAK3 and STAT6), leading to induction of E-cadherin/ $\beta$-catenin. ${ }^{9,13}$ Moreover, previous studies and results of the present study demonstrate that IL-4 regulates expression of numerous molecules including MCP-1, IL-10, MMP-9, and TNF- $\alpha$ via unknown pathways. ${ }^{10-12,42-44}$ Thus, in addition to IL-4-induced signals, it is appreciated that signals from the engagement

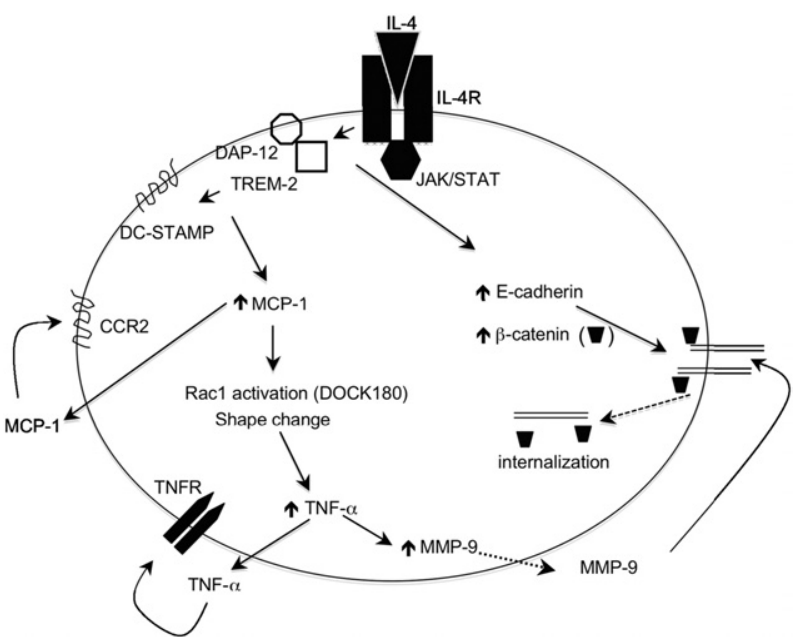

Figure 7. Model for the participation of MCP-1, TNF- $\alpha$, MMP-9, E-cadherin, and $\beta$-catenin in macrophage fusion. IL- 4 induces activation of at least two pathways including the JAK/STAT pathway (JAK1/JAK3 and STAT6), leading to induction of E-cadherin, $\beta$-catenin, MCP-1, TNF- $\alpha$, and MMP-9. Studies in DAP12-null macrophages suggest that DAP12 signaling is essential for programming macrophages into a fusion-competent state. MCP-1-null macrophages are deficient in cytoskeletal remodeling and have low levels of TNF- $\alpha$ and MMP-9 but induce E-cadherin and $\beta$-catenin normally. This implies that part of the JAK/STAT pathway is intact in MCP-1-null cells and that at least two divergent pathways are involved in fusion. Addition of TNF- $\alpha$ to MCP1-null macrophages in a fusion experiment induces MMP-9 expression and restores the fusion defect. It is suggested that a link between the two pathways exists based on the effects of MCP-1 and MMP-9 deficiency on the subcellular localization of E-cadherin and $\beta$-catenin.

of CCR2, TNFR1, and perhaps other receptors would integrate into multiple pathways. The present study demonstrated that despite being inherently deficient in fusion, MCP-1-null macrophages induce E-cadherin and $\beta$-catenin after IL-4 treatment. This implies that part of the JAK/STAT pathway is intact in MCP-1-null cells and that at least two divergent pathways are involved in fusion. Moreover, Rac1 inhibition prevents induction of MMP-9 but not $\mathrm{E}$-cadherin or $\beta$-catenin. Addition of $\mathrm{TNF}-\alpha$ restores induction of MMP-9 and the fusion defect in MCP1-null macrophages. It is suggested that a link between the two pathways exists, based on the effects of MCP-1 and MMP-9 deficiencies on the subcellular localization of E-cadherin and $\beta$-catenin. A recent study has implicated MT1-MMP, a membrane-bound MMP, in the process of fusion via interaction with the adaptor protein p130Cas, leading to regulation of Rac1 activity. ${ }^{45}$ However, integration of MT1-MMP signaling with signals propagated by IL-4 via DAP12, TREM-2, and DC-STAMP has not been defined.

In vitro and in vivo studies have demonstrated MMP9 -dependent redistribution of E-cadherin and $\beta$ catenin during fusion. Thus, it was postulated that redistribution of E-cadherin is a fusion-associated event, a suggestion that is in line with the ability of the E-cadherin/ $\beta$-catenin complex to regulate a number of cell processes including changes in cell shape, motility, and adhesion. ${ }^{46}$ The present results clearly demonstrate that induction of E-cadherin/ $\beta$-catenin in the absence of MCP-1-induced MMP-9 is not adequate to cause macrophage fusion. Thus, it is clear that fusion 
requires engagement of at least two independent pathways.

\section{Acknowledgments}

We thank Jianmin Zeng, B.Sc., for assistance with biomaterial implantation and Kristin Knox, B.Sc., and Sarah Tittman, B.Sc., for assistance with immunohistochemical staining.

\section{References}

1. Chen $\mathrm{EH}$, Olson EN: Unveiling the mechanisms of cell-cell fusion. Science 2005, 308:369-373

2. Oren-Suissa M, Podbilewicz B: Cell fusion during development Trends Cell Biol 2007, 17:537-546

3. Vignery A: Macrophage fusion: the making of osteoclasts and giant cells. J Exp Med 2005, 202:337-340

4. Helming L, Gordon S: Molecular mediators of macrophage fusion. Trends Cell Biol 2009, 19:514-522

5. Anderson JM, Rodriguez A, Chang DT: Foreign body reaction to biomaterials. Semin Immunol 2008, 20:86-100

6. Luttikhuizen DT, Harmsen MC, Van Luyn MJ: Cellular and molecular dynamics in the foreign body reaction. Tissue Eng 2006, 12:19551970

7. Yagi M, Miyamoto T, Sawatani $Y$, Iwamoto K, Hosogane N, Fujita N, Morita K, Ninomiya K, Suzuki T, Miyamoto K, Oike Y, Takeya M, Toyama Y, Suda T: DC-STAMP is essential for cell-cell fusion in osteoclasts and foreign body giant cells. J Exp Med 2005, 202:345351

8. Jay SM, Skokos E, Laiwalla F, Krady MM, Kyriakides TR: Foreign body giant cell formation is preceded by lamellipodia formation and can be attenuated by inhibition of Rac1 activation. Am J Pathol 2007, 171: $632-640$

9. Moreno JL, Mikhailenko I, Tondravi MM, Keegan AD: IL-4 promotes the formation of multinucleated giant cells from macrophage precursors by a STAT6-dependent, homotypic mechanism: contribution of E-cadherin. J Leukoc Biol 2007, 82:1542-1553

10. Helming L, Tomasello E, Kyriakides TR, Martinez FO, Takai T, Gordon $\mathrm{S}$, Vivier E: Essential role of DAP12 signaling in macrophage programming into a fusion-competent state. Sci Signal 2008, 1:ra11

11. Kyriakides TR, Foster MJ, Keeney GE, Tsai A, Giachelli CM, ClarkLewis I, Rollins BJ, Bornstein P: The CC chemokine ligand, CCL2/ MCP1, participates in macrophage fusion and foreign body giant cell formation. Am J Pathol 2004, 165:2157-2166

12. MacLauchlan S, Skokos EA, Meznarich N, Zhu DH, Raoof S, Shipley $\mathrm{JM}$, Senior RM, Bornstein P, Kyriakides TR: Macrophage fusion, giant cell formation, and the foreign body response require matrix metalloproteinase 9. J Leukoc Biol 2009, 85:617-626

13. Van den Bossche J, Bogaert P, van Hengel J, Guerin CJ, Berx G, Movahedi K, Van den Bergh R, Pereira-Fernandes A, Geuns JM, Pircher H, Dorny P, Grooten J, De Baetselier P, Van Ginderachter JA: Alternatively activated macrophages engage in homotypic and heterotypic interactions through IL-4 and polyamine-induced E-cadherin/catenin complexes. Blood 2009, 114:4664-4674

14. Gu L, Tseng SC, Rollins BJ: Monocyte chemoattractant protein-1. Chem Immunol 1999, 72:7-29

15. Conti I, Rollins BJ: CCL2 (monocyte chemoattractant protein-1) and cancer. Semin Cancer Biol 2004, 14:149-154

16. Lu B, Rutledge BJ, Gu L, Fiorillo J, Lukacs NW, Kunkel SL, North R, Gerard C, Rollins BJ: Abnormalities in monocyte recruitment and cytokine expression in monocyte chemoattractant protein 1-deficient mice. J Exp Med 1998, 187:601-608

17. Kurihara T, Warr G, Loy J, Bravo R: Defects in macrophage recruitment and host defense in mice lacking the CCR2 chemokine receptor. J Exp Med 1997, 186:1757-1762

18. Kuziel WA, Morgan SJ, Dawson TC, Griffin S, Smithies O, Ley K, Maeda N: Severe reduction in leukocyte adhesion and monocyte extravasation in mice deficient in CC chemokine receptor 2. Proc Natl Acad Sci USA. 1997, 94:12053-12058

19. Mendes JB, Campos PP, Ferreira MA, Bakhle YS, Andrade SP: Host response to sponge implants differs between subcutaneous and intraperitoneal sites in mice. J Biomed Mater Res B Appl Biomater 2007, 83:408-415

20. Jay SM, Skokos EA, Zeng J, Knox K, Kyriakides TR: Macrophage fusion leading to foreign body giant cell formation persists under phagocytic stimulation by microspheres in vitro and in vivo in mouse models. J Biomed Mater Res A 2010, 93:189-199

21. Brodbeck WG, Nakayama Y, Matsuda T, Colton E, Ziats NP, Anderson JM: Biomaterial surface chemistry dictates adherent monocyte/ macrophage cytokine expression in vitro. Cytokine 2002, 18:311-319

22. Henderson RB, Hobbs JA, Mathies M, Hogg N: Rapid recruitment of inflammatory monocytes is independent of neutrophil migration. Blood 2003, 102:328-335

23. van Roy F, Berx G: The cell-cell adhesion molecule E-cadherin. Cell Mol Life Sci 2008, 65:3756-3788

24. Delva E, Kowalczyk AP: Regulation of cadherin trafficking. Traffic 2009, 10:259-267

25. Saren P, Welgus HG, Kovanen PT: TNF-alpha and IL-1beta selectively induce expression of $92-\mathrm{kDa}$ gelatinase by human macrophages. J Immunol 1996, 157:4159-4165

26. Zhang Y, McCluskey K, Fujii K, Wahl LM: Differential regulation of monocyte matrix metalloproteinase and TIMP-1 production by TNFalpha, granulocyte-macrophage CSF, and IL-1 beta through prostaglandin-dependent and -independent mechanisms. J Immunol 1998, 161:3071-3076

27. Vaday GG, Schor H, Rahat MA, Lahat N, Lider O: Transforming growth factor-beta suppresses tumor necrosis factor alpha-induced matrix metalloproteinase-9 expression in monocytes. J Leukoc Bio 2001, 69:613-621

28. Gordon S, Martinez FO: Alternative activation of macrophages: mechanism and functions. Immunity 32:593-604

29. Ferreira AM, Rollins BJ, Faunce DE, Burns AL, Zhu X, Dipietro LA: The effect of MCP-1 depletion on chemokine and chemokine-related gene expression: evidence for a complex network in acute inflammation. Cytokine 2005, 30:64-71

30. Mosser DM, Edwards JP: Exploring the full spectrum of macrophage activation. Nat Rev Immunol 2008, 8:958-969

31. Daley JM, Brancato SK, Thomay AA, Reichner JS, Albina JE: The phenotype of murine wound macrophages. J Leukoc Biol 87:59-67

32. Jones JA, McNally AK, Chang DT, Qin LA, Meyerson H, Colton E, Kwon IL, Matsuda T, Anderson JM: Matrix metalloproteinases and their inhibitors in the foreign body reaction on biomaterials. J Biomed Mater Res A 2008, 84:158-166

33. Okuma T, Terasaki Y, Kaikita K, Kobayashi H, Kuziel WA, Kawasuji M, Takeya M: C-C chemokine receptor 2 (CCR2) deficiency improves bleomycin-induced pulmonary fibrosis by attenuation of both macrophage infiltration and production of macrophage-derived matrix metalloproteinases. J Pathol 2004, 204:594-604

34. Tang L, Eaton JW: Natural responses to unnatural materials: a molecular mechanism for foreign body reactions. Mol Med 1999, 5:351-358

35. Shive MS, Brodbeck WG, Colton E, Anderson JM: Shear stress and material surface effects on adherent human monocyte apoptosis. J Biomed Mater Res 2002, 60:148-158

36. Robinson SC, Scott KA, Balkwill FR: Chemokine stimulation of monocyte matrix metalloproteinase-9 requires endogenous TNF-alpha. Eur $\mathrm{J}$ Immunol 2002, 32:404-412

37. Rhee JW, Lee KW, Sohn WJ, Lee Y, Jeon OH, Kwon HJ, Kim DS: Regulation of matrix metalloproteinase-9 gene expression and cell migration by NF-kappa B in response to CpG-oligodeoxynucleotides in RAW 264.7 cells. Mol Immunol 2007, 44:1393-1400

38. Bianchi K, Meier P: A tangled web of ubiquitin chains: breaking news in TNF-R1 signaling. Mol Cell 2009, 36:736-742

39. Lee IT, Lin CC, Wu YC, Yang CM: TNF-alpha induces matrix metalloproteinase-9 expression in A549 cells: role of TNFR1/TRAF2/PKCalpha-dependent signaling pathways. J Cell Physiol 224:454-464

40. Hotokezaka H, Sakai E, Ohara N, Hotokezaka Y, Gonzales C, Matsuo K, Fujimura Y, Yoshida N, Nakayama K: Molecular analysis of RANKL-independent cell fusion of osteoclast-like cells induced by TNF-alpha, lipopolysaccharide, or peptidoglycan. J Cell Biochem 2007, 101:122-134

41. Symowicz J, Adley BP, Gleason KJ, Johnson JJ, Ghosh S, Fishman DA, Hudson LG, Stack MS: Engagement of collagen-binding integrins promotes matrix metalloproteinase-9-dependent E-cadherin ectodomain shedding in ovarian carcinoma cells. Cancer Res 2007, 67:2030-2039 
42. Varin A, Mukhopadhyay S, Herbein G, Gordon S: Alternative activation of macrophages by IL-4 impairs phagocytosis of pathogens but potentiates microbial-induced signalling and cytokine secretion. Blood 115:353-362

43. Chomarat P, Rissoan MC, Banchereau J, Miossec P: Interferon gamma inhibits interleukin 10 production by monocytes. J Exp Med 1993, 177:523-527

44. Bonder CS, Finlay-Jones JJ, Hart PH: Interleukin-4 regulation of human monocyte and macrophage interleukin-10 and interleukin-12 production: role of a functional interleukin-2 receptor gamma-chain. Immunology 1999, 96:529-536

45. Gonzalo P, Guadamillas MC, Hernandez-Riquer MV, Pollan A, Grande-Garcia A, Bartolome RA, Vasanji A, Ambrogio C, Chiarle R, Teixido J, Risteli J, Apte SS, del Pozo MA, Arroyo AG: MT1-MMP is required for myeloid cell fusion via regulation of Rac1 signaling. Dev Cell 18:77-89

46. Nelson WJ: Regulation of cell-cell adhesion by the cadherin-catenin complex. Biochem Soc Trans 2008, 36:149-155 